\title{
Neuroprotective Effect of Rosuvastatin on ca1 \& ca3 Regions of Hippocampus in High Fat Diet and Stress induced Rats
}

\author{
Rajesh Vaderav $^{1 *}$, Subadhradevi Velichety ${ }^{2}$, Chandramohan Madabhushi ${ }^{1}$, Susheelamma D ${ }^{1}$
}

${ }^{1}$ Department of Anatomy, Mamata Medical College, Khammam, India

${ }^{2}$ Department of Anatomy, Sri Venkateswara Institute of Medical Sciences, Tirupati, India

\begin{abstract}
Back ground: Prolonged stress and diet rich in cholesterol is known to cause memory impairment and cause disruption of neuronal net work in hippocampus. In the present study, we evaluated the neuroprotective effect of rosuvastatin HMG CoA reductase inhibitor on stress induced and high fat diet fed rats given in combination.

Materials \& methods: Forty eight adult male wistar rats were randomly assigned into eight groups. ( $\mathrm{N}=6)$ control group received normal diet (chow diet), second group received only high fat diet (HFD), third group received only stress (STS), fourth group high fat diet and stress (HFD+STS), fifth group control + rosuvastatin $10 \mathrm{mg} /$ body wt, sixth group control + rosuvastatin $20 \mathrm{mg} / \mathrm{body}$.wt, seventh group received high fat diet + stress and treated with rosuvastatin10mg/body.wt (HFD+STS+ROS 10mg), eighth group was treated with rosuvastatin 20mg/body.wt (HFD+STS+ROS 20mg).Spatial memory was assessed by morris water maze. Rats were sacrificed at $96^{\text {th }}$ day; brains were removed and processed for histological studies using cresyl violet staining. Neuronal population of CA1 \&CA3 region was quantified.

Results: A significant $(\mathrm{p}<0.01)$ increase in the neuronal population in the sub regions of hippocampus and improvement of spatial memory (CA1:32 \pm 1.95 , CA3: $22 \pm 1.5$, latency to enter the target quadrant LT: $6.05 \pm 0.9 \mathrm{sec}$, time spent in target quadrant TST: $14.4 \pm 1.62 \mathrm{sec}$ was seen in rats treated with rosuvastatin $20 \mathrm{mg} / \mathrm{kg}$.body.wt (HFD+STS+ROS $20 \mathrm{mg}$ ) compared to high fat diet and stress (HFD+STS): (CA1: $28 \pm 1.33, \mathrm{CA} 3: 18 \pm 1.47 \&$ LT: $7.67 \pm 0.77 \mathrm{sec}, \mathrm{TST}: 10 \pm 1.37 \mathrm{sec}$. The rats treated with $10 \mathrm{mg} / \mathrm{kg} . \mathrm{body}$. wt (CA1:30 $\pm 1.83, \mathrm{CA} 3: 22 \pm 1.37)$ did not show any significance compared to HFD+STS group.

There was also significant $(\mathrm{p}<0.01)$ improvement in only high fat group treated with rosuvastatin $20 \mathrm{mg} / \mathrm{kg}$.body.wt (HFD+ROS20mg) CA1:33.7 \pm 1.37 , CA3: 26.7 \pm 1.2 , LT: $3.31 \pm 0.4 \mathrm{sec}$, TST: $24.5 \pm 1.77 \mathrm{sec}$, compared to (HFD) : CA1:30 \pm 2.04 , CA3: 241.9, LT: $4.66 \pm 0.84 \mathrm{sec}$, TST: $2.46 \pm 1 \mathrm{sec}$.

Conclusion: The results clearly demonstrate that rosuvastatin $20 \mathrm{mg} / \mathrm{kg}$.body.wt was able to prevent hippocampal neuronal loss in CA1 and CA 3 regions and also enhanced learning and memory abilities. These findings support the view that rosuvastatin have neuroprotective action.
\end{abstract}

Keywords: Hippocampus; Rosuvastatin; Morris water maze

Corresponding author: Rajesh Vaderav, Department of Anatomy, Mamata Medical College, Khammam, India, E-mail: rajeshvaderav@gmail.com

Citation: : Rajesh, V., et al. Neuroprotective Effect of Rosuvastatin on Ca1 \& Ca3 Regions of Hippocampus in High Fat Diet and Stress Induced Rats. (2014) Int J Neurol Brain Disord 1(1): 19- 23.

\section{Received Date: Nov 27, 2014}

\section{Introduction}

Cholesterol is the main constituent of the high fat diet. Pre-clinical studies have shown that dietary cholesterol can be the cause for neurodegenerative diseases and dementia (Alzeheimer's disease) ${ }^{[1,2]}$. Animal studies showed that high fat diet impairs hippocampal neurogenesis in male wistar rats ${ }^{[3]}$ and epidemiological studies support the view that dietary cholesterol is one of the causes for Alzheimer's disease and vascular dementia $^{[4]}$.

Prolonged stress alters the possible mechanisms like hypothalamo-pituitary-adrenal axis, neurotransmitters, decreases brain cell proliferations and brain corticosterone level during prenatal development ${ }^{[5]}$. Animal studies and clinical studies have demonstrated the stress effects cognitive tasks and it is one of the risk factor for dementia. Reports have suggested that chron-

\section{Published Date: Dec 31, 2014}

ic stress have reduced BDNF(brain derived neurotropic factor) values $^{[6]}$. Recent findings suggested that combination of high fat diet and stress have produced extensive atrophy of dendrites of pyramidal neurons in hippocampal area ${ }^{[7]}$.

Rosuvastatin is a hypolipidemic drug (statin) which acts by inhibiting rate limiting HMG-COA reductase. Recent reports suggest that rosuvastatin exert additional pleiotropic activities that are independent of its hypolipidemic activity which includes antioxidant activity, anti-inflammatory activity, improvement of endothelial function, and stabilization of atherosclerotic plaques ${ }^{[8]}$. These findings have led to the speculation that rosuvastatin may have potential therapeutic implications in various neurological disorders. Investigations addressing the relationship between dementia and statins are mixed. Findings from cross-sectional studies suggested a protective role for statins in dementia but few prospective designed and cohort

Copy rights: (C2014 Rajesh, V. This is an Open access article distributed under the terms of Creative Commons Attribution 4.0 International License. 
studies, showed conflicting results ${ }^{[9,10]}$.

\section{Aims and Objectives}

The aim of the present study is to explore the neuroprotective action of rosuvastatin in CA1 and CA3 regions of hippocampus when high fat diet and prolonged stress given to rats in combination.

\section{Materials and methods}

\section{Animals}

Inbred male Wistar strain weighing 120-150g were selected and procured from central animal house, Mamata Medical College Khammam. Six rats were housed in propylene cages $(22.5 \times 35.5 \times 15 \mathrm{~cm})$ maintained in 12 hours light and dark cycle in temperature and humidity controlled environment, and were fed with standard food pellet and water ad libitum. The experimental protocol was approved by the institutional animal ethical committee. (IAEC/DP-05/C16).All the experimental procedures were carried out during 10 am to $4 \mathrm{pm}$.

Animals were randomly assigned into three groups of seven each.

Group 1: (control) Fed with standard chow diet for three months. Group 2: Fed with high fat diet for three months (HFD)

Group 3: Fed with high fat diet and received stress for 21days (HFD + STS)

Group 4: Fed with high fat diet and treated with $10 \mathrm{mg} / \mathrm{kg}$.body. wt (HFD + ROS $10 \mathrm{mg}$ )

Group 5: Fed with high fat diet and treated with $20 \mathrm{mg} / \mathrm{kg}$.body. wt (HFD + ROS $20 \mathrm{mg}$ )

Group 6: Fed with high fat diet and received stress and treated with rosuvastatin $10 \mathrm{mg} / \mathrm{kg}$.body.wt (HFD + STS + ROS $10 \mathrm{mg}$ ) Group 7: Fed with high fat diet and received stress and treated with rosuvastatin $20 \mathrm{mg} / \mathrm{kg}$.body.wt (HFD + STS + ROS $20 \mathrm{mg}$ )

Method of inducing hyperlipidemia: Hyperlipidemia has been induced by feeding with cholesterol-rich diet for 3 months. Deoxycholic acid $(5 \mathrm{~g})$ was mixed thoroughly with $700 \mathrm{~g}$ of powdered rat chow diet. Simultaneously cholesterol (5g) was dissolved in $300 \mathrm{~g}$ warm coconut oil. This oily solution was added slowly into the powdered mixture to obtain a soft homogenous cake. This cholesterol-rich diet was made into pellets of about $3 \mathrm{~g}$ each and given to the animals ${ }^{[1]]}$.

Induction of stress to the rat's protocol: Male wistar rats were placed in a wire mesh restrainer for 21 days, daily 6 hours, from $69^{\text {th }}$ to $90^{\text {th }}$ day. The restrainer was made up of a wooden base to which stainless steel wire mesh was hinged. A pad lock and latch were used to secure the rat. The dimensions were $8 \mathrm{~cm}$ (Length) x $4 \mathrm{~cm}$ (Breadth) $\times 4 \mathrm{~cm}$ (Height). This type of wire mesh restrainer can only restrict the animal movement without any uneasiness, pain or suffocation ${ }^{[12]}$.

Treatment with rosuvastatin: Rosuvastatin was obtained from Pfiger Company Mumbai. Groups 5,6,7,8rats were treated with $10 \mathrm{mg} / \mathrm{kg}$ body weight and $20 \mathrm{mg} / \mathrm{kg}$ body weight rosuvastatin for 14 days from $76^{\text {th }}-90^{\text {th }}$ day ${ }^{[13]}$.

Morris water maze test: Spatial memory of the rats was tested on $90^{\text {th }}$ day by using Morris water maze. The water maze consists of a circular tank of $1.80 \mathrm{~m}$ diameter and $75 \mathrm{~cm}$ depth. The pool was filled with water and maintained at a temperature of $24-26^{\circ} \mathrm{C}$ to a depth of about $50 \mathrm{~cm}$. It was divided into four quadrants and an escape platform of size 4 " $\times 4$ " was hidden approximately $2 \mathrm{~cm}$ below the water surface in the target quadrant. Water in the pool was made opaque by adding milk just before the experiment. Permanently positioned distinctive objects were placed for facilitating spatial orientation of the animal. Positions of the cues were kept unchanged throughout the period of experiment. The rats were trained in the water maze in 10 sessions on 5 consecutive days, two sessions on each day. Each session consists of four trails. In each trail time taken to reach hidden platform was recorded. If the rat was unable to find the platform within two minutes, the training session was terminated and a maximum score of two minutes was assigned.

Twenty four hours after the last session, rats were subjected to a probe trail. This session was for 30 in which the hidden platform was removed. Here time taken to reach the target quadrant and time spent by the rats in search of the platform was measured. Greater latency to reach the target quadrant and less time to spend in the target quadrant suggests memory impairment $^{[14]}$.

Weight gain Weight of the animals was measured at the beginning of the study and at the end (after 3 months) to calculate the net weight gained.

Lipid profile Blood samples were obtained by retro-orbital puncture, serum cholesterol and triglycerides were estimated on $96^{\text {th }}$ day ${ }^{[15]}$.

\section{Cresyl violet staining procedure}

The animals were profoundly anesthetized with ether and fixed to dissection board. The chest cavity was opened and heart was exposed. About $15 \mathrm{ml}$ of $0.9 \%$ heparinized saline was perfused through left ventricle at the rate of $1 \mathrm{ml} / \mathrm{min}$ followed by $250 \mathrm{ml}$ of $10 \%$ formalin. The animal was decapitated and the brain was isolated. $5-6 \mathrm{~mm}$ thick coronal sections of brain tissue were made and kept in $10 \%$ formalin for 48 hours (post fixation). Paraffin blocks were made and sections of 5 microns thickness were cut from the mid dorsal hippocampal level, using a rotary microtome. Sections were selected and mounted serially on air dried gelatinized slides. Tissues were processed through different grades of alcohol $(50 \%, 70 \%$ for $24 \mathrm{~h}, 90 \%$, and $100 \%$ for 12 $\mathrm{h}$ ) and were immersed in xylene for 1-2 $\mathrm{h}$.

\section{Preparation of Cresyl violet stain (0.1\%)}

$100 \mathrm{mg}$ of Cresyl violet was dissolved in $100 \mathrm{ml}$ of distilled water. To this $0.5 \mathrm{ml}$ of $10 \%$ Acetic acid was added to give a $\mathrm{pH}$ of 3.5 to 3.8.The stain was filtered before use.

\section{Quantification of cells}

In each hippocampal section, cornua amonis(CA1 and CA3; $250 \mu \mathrm{m}$ length) was selected using an oculomicrometer. The number of viable neurons were counted and averaged under (40X) magnification (Magnus, Olympus Pvt. Ltd. New Delhi, India). Darkly stained, shrunken and cells with fragmented nuclei were excluded from the count. To avoid manual bias ten sections from each rat were considered. The cell counts were expressed as the number of cells per unit length of the cell field $(\text { cells } / 250 \mu \mathrm{m})^{[16]}$. 


\section{Statistical analysis}

The data were expressed as mean $\pm \mathrm{SE}$. The significant differences among the groups were assessed using one way analysis of Variance (ANOVA) followed by Bonferroni multiple comparison test in Graph Pad in Stat (GPIS) software, version 1.13. $\mathrm{p}$ values $<0.05$ were considered as significant

\section{Results and Statistical Analysis}

Weight of the animals was measured during the study period (before and after three months). There was significant increase in the body weight of the animals fed with only high fat diet (HFD: $264 \pm 6.90,(\mathrm{p}<0.001)$ and high fat diet + stress (HFD+STS: 172 $\pm 9.61(\mathrm{p}<0.01)$, when compared to control group animals $(\mathrm{C}$ : $149 \pm 7.36)$.

Significant decrease in body weight was observed in rats treated with rosuvastatin $10 \mathrm{mg}$ (HFD+STS+ROS $10 \mathrm{mg}$ / kg.body.wt:152 \pm 3.76 , HFD+ROS10mg/kg.body.wt:243 \pm 7.08 $\mathrm{p}<0.01)$ compared to HFD+STS group and HFD groups. In rats treated with $20 \mathrm{mg}$ rosuvastatin also there was very significant decrease in body weights (HFD+STS+ROS 20mg/kg.body. wt: $145 \pm 4.47$, HFD+ROS20mg/kg.body.wt:235 \pm 13.8 p $<0.001)$ compared to (HFD+STS and HFD groups).

After three months serum cholesterol (cho) and triglyceride (tri) levels were elevated in both groups namely high fat diet (HFD) group (cho: $166 \pm 4.64$, tri: $149 \pm 6.31),(\mathrm{p}<0.001)$ and HFD + STS (cho: 92.2 \pm 3.43 , tri: $101 \pm 5.85),(\mathrm{p}<0.01)$ as compared to the control group (cho: $80.7 \pm 5.79$, tri: $88.2 \pm 5.96$ ). In groups treated with rosuvastatin $20 \mathrm{mg} / \mathrm{kg}$.body.wt and $10 \mathrm{mg} /$ kg.body.wt there was significant decrease in cholesterol and triglycerides. In rosuvastatin $10 \mathrm{mg}$ treated groups the significance was $\mathrm{p}<0.01$ (HFD+ROS10mg/kg.body.wt: cho $154 \pm 4.92$, tri 137.8 \pm 5.12 , HFD+STS+ROS10mg/kg.body.wt cho $82.3 \pm 2.7$, tri: $88.3 \pm 4.08$ ) compared HFD and HFD+STS group. In Rosuvastatin $20 \mathrm{mg}$ treated groups the significance was $\mathrm{p}<0.001$ (HFD+ROS20mg/kg.body.wt:cho139 \pm 3.76 , tri $124.8 \pm 4.5$, HFD+STS+ROS20mg/kg.body.wt cho $80.5 \pm 6.92$, tri: $85.5 \pm 3.39$ ) compared to HFD and HFD+STS groups.

\section{Behavioural test (Morris water maze): \\ Latency to enter the target quadrant}

This was done on $96^{\text {th }}$ day, twenty four hours after the 10th training session. Compared to control group (C: $3.32 \pm 0$ $.76 \mathrm{sec}$, high fat diet group HFD: $4.66 \pm 0.84 \mathrm{sec}$ took longer time $(\mathrm{p}<0.05)$ and high fat diet plus stress group HFD+STS: $7.67 \pm 0.77 \mathrm{sec}$ took significantly $(\mathrm{p}<0.001)$ more time to reach the target quadrant (probe) suggesting memory impairment.

Rats treated with $10 \mathrm{mg}$ rosuvastatin took (HFD+ROS$10 \mathrm{mg} / \mathrm{kg}$.body.wt) $3.95 \pm 0.75 \mathrm{sec}$ and (HFD+STS+ROS $10 \mathrm{mg} /$ kg.body.wt) $7.07 \pm 0.56 \mathrm{sec}$ which did not show any significance $(p<0.01)$ significantly less time to enter the target quadrant (HFD+ROS20mg/kg.body.wt) $3.31 \pm 0.4 \mathrm{sec}$ and (HFD+STS+ROS $20 \mathrm{mg} / \mathrm{kg}$.body.wt) $6.05 \pm 0.91 \mathrm{sec}$ compared to HFD+STS group The above results imply that only rosuvastatin given in higher dose $20 \mathrm{mg}$ have improved the spatial memory. (Figure 1)

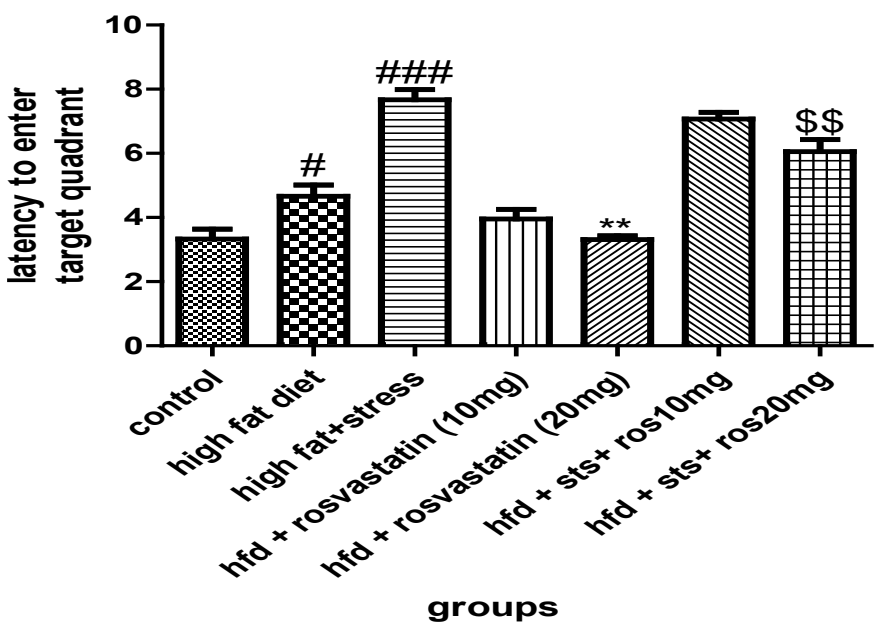

Figure 1: Bar graph showing the latency to enter target quadrant (sec). Bars represent mean \pm SEM (control vs HFD + STS $^{\# \#} \mathrm{p}<0.001$, control vs HFD ${ }^{\#} \mathrm{p}$ $<0.05)\left(\mathrm{HFD}+\mathrm{ROS} 20 \mathrm{mg}\right.$ vs $\left.\mathrm{HFD}^{* *} \mathrm{p}<0.01\right)(\mathrm{HFD}+\mathrm{STS}+\mathrm{ROS} 20 \mathrm{mg}$ vs $\left.\mathrm{HFD}+\mathrm{STS}^{\mathrm{ss}} \mathrm{p}<0.01\right)$.

\section{Time spent in the target quadrant}

High fat diet plus stress induced rats (HFD+STS: 10 $\pm 1.37 \mathrm{sec})$ spent significantly $(\mathrm{p}<0.001)$ lesser amount of time and rats fed with only high fat fed group took less time ( $\mathrm{p}<0.05$, HFD: $20.2 \pm 2.46 \mathrm{sec}$ ) in the target quadrant (probe) in search of the missing platform compared to control group (C: $24 \pm$ $3.01 \mathrm{sec}$ ). This shows that both stress and high fat diet in combination produces more memory deficits.

The spatial memory of rats treated with rosuvastatin $20 \mathrm{mg}$ was significantly improved in (HFD+STS+ROS $20 \mathrm{mg} /$ kg.body.wt $\mathrm{p}<0.01,14.3 \pm 1.62 \mathrm{sec})$ and took longer time in search of the missing platform but (HFD+STS+ROS: $10 \mathrm{mg}$ $13.2 \pm 1.51 \mathrm{sec})$ group did not show any difference compared to the high fat diet plus stress group HFD+STS.

The spatial memory of the rats treated with $20 \mathrm{mg}$ rosuvastatin in HFD +ROS $20 \mathrm{mg} / \mathrm{kg}$.body.wt $24.5 \pm 1.77 \mathrm{sec}$ took significantly less time $\mathrm{p}<0.01$ but rats treated with $10 \mathrm{mg}$ (HFD+ROS $10 \mathrm{mg} / \mathrm{kg}$.boy.wt $19.1 \pm 2.17 \mathrm{sec}$ ) did not show any significance compared to HFD group. This shows that rosuvastatin given in higher dose enhances spatial memory. (Figure 2)

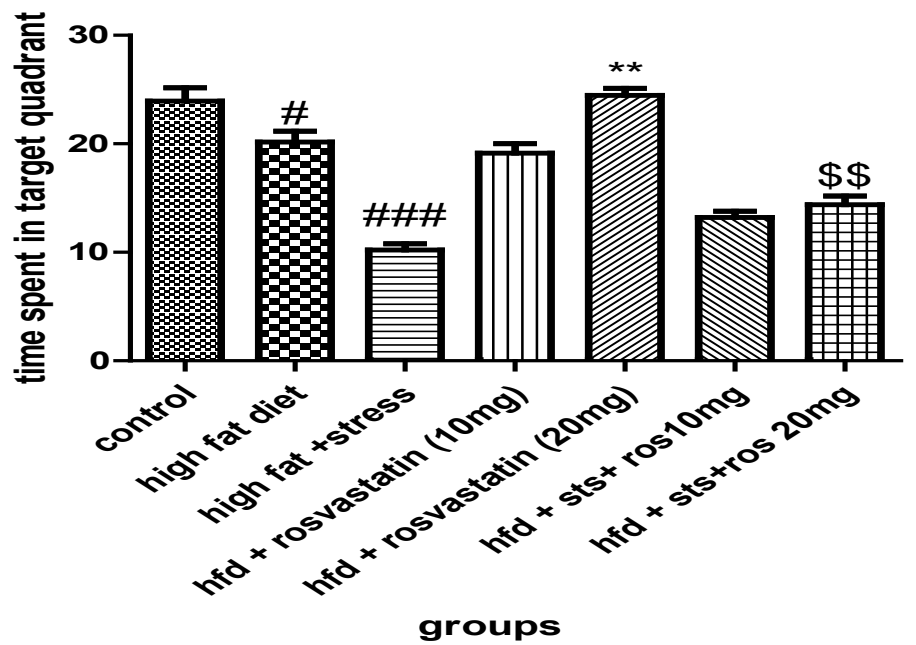

Figure 2: Bar graph showing the time spent in target quadrant (sec). Bars represent mean \pm SEM (control vs HFD + STS $^{\# \#} \mathrm{p}<0.001$, control vs $\mathrm{HFD}^{\#} \mathrm{p}<0.05$ )

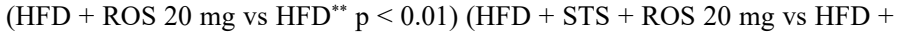
STS $\left.^{\$ \$} \mathrm{p}<0.01\right)$. 


\section{Histology hippocampus (cresyl violet staining)}

The number of viable neurons of CA1 and CA 3 regions of hippocampus showed significant decrease in High fat diet + stress group (HFD+STS: CA1:28 \pm 1.33, CA3:18 $1.47, \mathrm{p}<0.001)$ and only high fat diet fed group (HFD: $30.2 \pm 2.04,24 \pm 1.97 \mathrm{p}<0.05$ ) compared to control group (C: $34 \pm 1.47,27 \pm 0.98$ ).

The number of viable neurons in CA1 and CA3 regions in the rats treated with rosuvastatin $20 \mathrm{mg}$ group (HFD+STS+ROS 20mg/kg.body.wt:CA1:31 \pm 1.75 , CA3: 22 \pm 2.12 ; HFD+R0S20mg/kg.body.wt: CA1: $30 \pm 2.04$, CA3:26.7 \pm 1.21 $(\mathrm{p}<0.01)$ has significantly increased when compared to (HFD+STS) and HFD groups. The group which was treated with rosuvastatin $10 \mathrm{mg}$ (HFD+STS+ROS10mg/kg.body.wt CA1:29 \pm 0.33, CA3: $20 \pm$ 0.44) (HFD+ROS10mg/kg.body.wt: CA1:30 $\pm 1.8, C A 3: 22.3 \pm 1.37$ ) did not show any significance when compared with HFD+STS group and HFD group. This implies the neuroprotective action of rosuvastatin in higher dosage 20mg/kg.body.wt (Figure 3, 4, 5 \& 6).

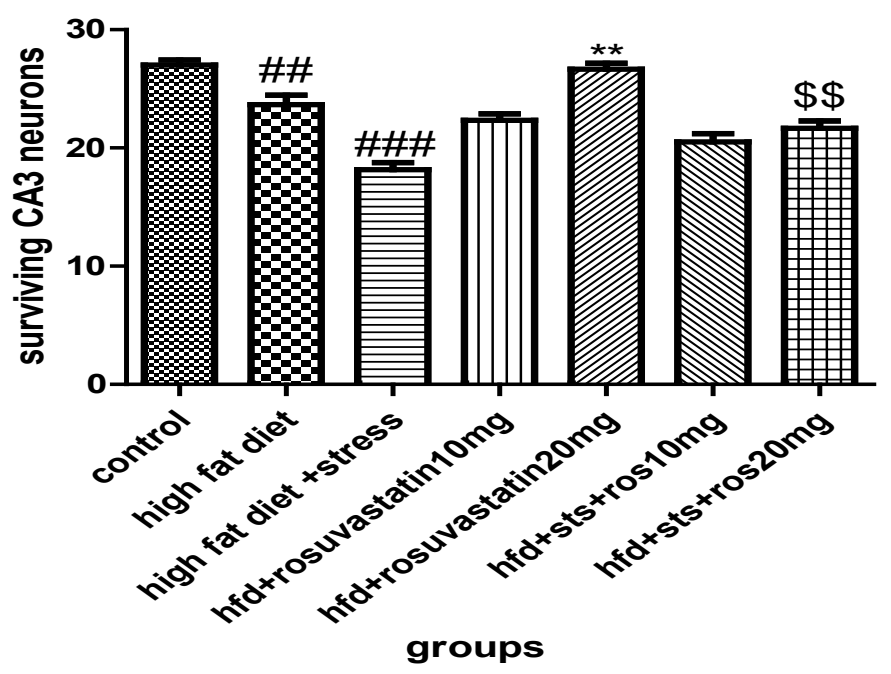

Figure 3: Bar graph showing the no of surviving neurons in $\mathrm{CA} 3$ region of hippocampus. Bars represent mean \pm SEM (control vs HFD + STS \#\# $\mathrm{p}<0.001$, control vs HFD ${ }^{\#}$ p $\left.<0.01\right)\left(H F D+\right.$ ROS 20 mg vs HFD ${ }^{* *}$ p $\left.<0.01\right)(H F D+S T S$ + ROS $20 \mathrm{mg}$ vs HFD + STS $\left.^{\text {\$\$ }} \mathrm{p}<0.01\right)$.

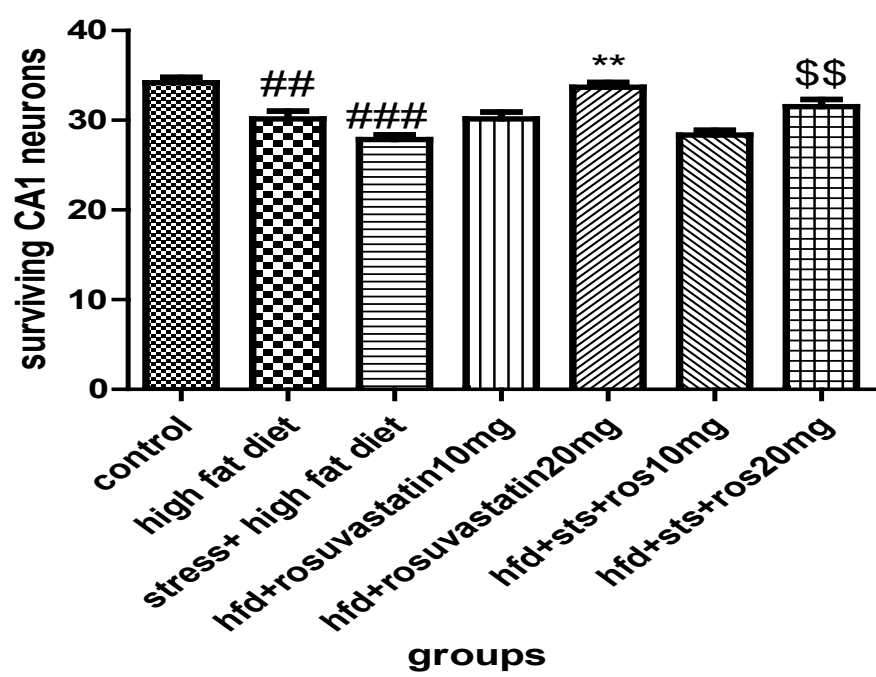

Figure 4: Bar graph showing the no of surviving neurons in CA1 region of hippocampus. Bars represent mean \pm SEM (control vs HFD $+\mathrm{STS}^{\#} \mathrm{p}<0.001$, control vs HFD ${ }^{\#} \mathrm{p}<0.01$ ) (HFD + ROS 20 mg vs HFD $\left.^{* *} \mathrm{p}<0.01\right)(\mathrm{HFD}+\mathrm{STS}$ + ROS 20 mg vs HFD + STS $\left.^{\$ \$} \mathrm{p}<0.01\right)$.

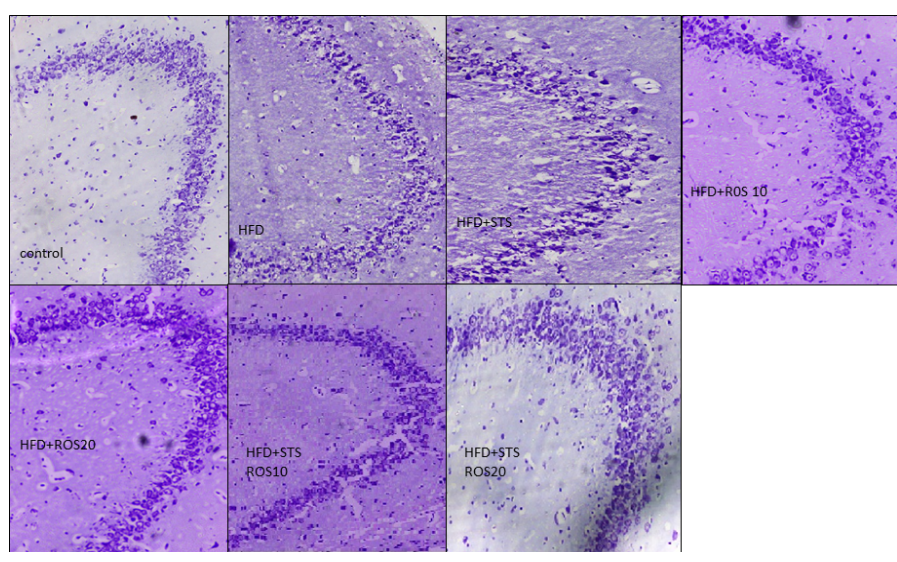

Figure 5 (Cresyl violet staining under 10X magnification. Coronal section of CA3 region of the hippocampus. Control vs HFD + STS $p<0.001$, control vs HFD p < 0.01) (HFD+ROS 20mg vs HFDp < 0.01) (HFD+ STS +ROS 20mg vs HFD + STSp $<0.01)$.

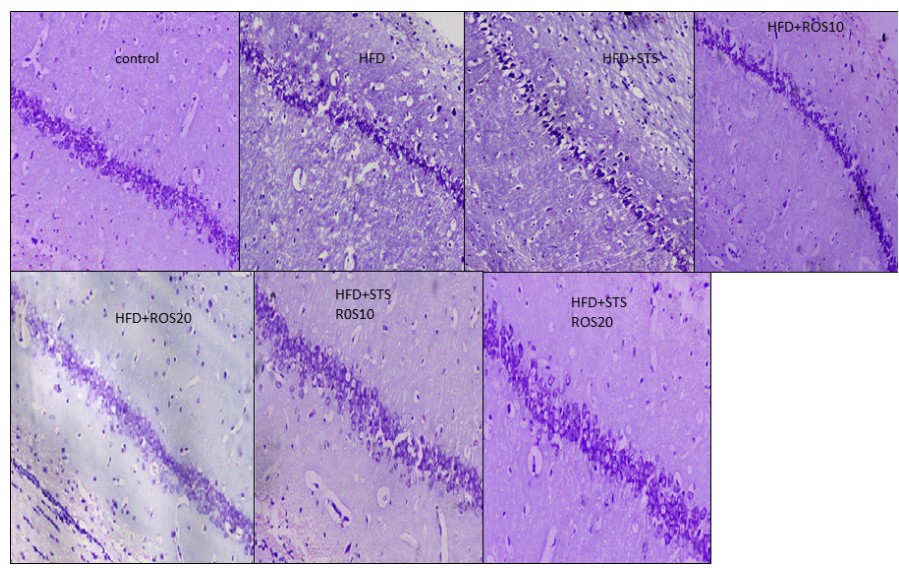

Figure 6 (Cresyl violet staining under 10X magnification). Coronal section of CA1 region of the hippocampus. (control vs HFD+STS $\mathrm{p}<0.001$, control vs HFD p < 0.01) (HFD+ROS 20mg vs HFD p < 0.01) (HFD+ STS +ROS 20mg vs HFD + STS $\mathrm{p}<0.01)$.

\section{Discussion}

The present study indicates that rosuvastatin in higher dosage 20mg has neuroprotective action on the neurons of cal and ca3 regions of hippocampus when compared to rats fed with high dietary fat and induced to stress.

In behavioural models (Morris water maze) a marked decrease in escape latency, as compared to first exposure denotes normal learning ability. Increase in the time spent by the animal in search of the missing platform in the target quadrant and decrease in time taken to reach the target quadrant gives us the successful retention of learned memory. In the present study rats treated with rosuvastatin $20 \mathrm{mg}$ has taken less time to reach the target quadrant and spent significantly more time in the target quadrant in the search of the missing platform compared to the high fat diet plus stress induced group.

Though there was a marked increase in the body weight of the rats which were given high fat diet (90 days) but there was no change in the swimming ability of the rats (driving motivation) in the rats compared to the control group rats.

Statins, which are 3-hydroxy-methylglutaryl coenzyme A reductase (HMG-CoA reductase) inhibitors, have been clinically used for treatment of hyperlipidemia. It has been suggested that the pleiotrophic effects of statins are responsible for their 
neuroprotective action. Gavin $\mathrm{j}$ blake et al stated that statins have anti inflammatory properties ${ }^{[17]}$. Broncel $M$ showed that statins have antioxidant properties and was useful in patients with dyslipidemia ${ }^{[18]}$.

Parle et al. have reported that atorvastatin and simvastatin have improved the spatial learning in rats treated with scopolamine ${ }^{[19]}$. Wolzian et al stated that, the prevalence of Alzheimer's disease has been lowered in the patients taking lovastatin or pravastatin ${ }^{[20]}$. Rech et al. found that rosuvastatin reduced the oxidative damage in the hippocampus and improved short-term memory in rats that received iron in the neonatal period ${ }^{[21]}$.

There was no difference in the neuroprotective properties between rosuvastatin(hydrophilic cannot cross the blood brain barrier) and atorvastain (lipophilic) ${ }^{[22]}$. Recent studies on transgenic rats, zucker obese rats have shown that treatment with rosuvastatin have improved cerebral vascular responsiveness and they were restored to normal ${ }^{[23]}$. Present study on male wistar rats is in line with the previous studies that support the hypothesis that statins have neuroprotective effect ${ }^{[24-26]}$.

\section{Conclusion}

Cure of cognitive disorders specially, dementias; is still a night mare in the field of medicine. Cholinesterase inhibitors, if used early in the course of disease can delay the progress of disease. Today, there is growing evidence that statins have several potentially beneficial effects by mechanisms unrelated to changes of cholesterol metabolism. However, we still know very little about the clinical relevance of this lipid independent statin properties. This study, is establishing the advantage of controlling hyperlipidemia, which in turn protects from memory impairment and further broaden the use of lipid lowering agents.

\section{References}

1. Wu, A., Molteni, R., Ying, Z., et al. A saturated-fat diet aggravates the outcome of traumatic brain injury on hippocampal plasticity and cognitive function by reducing brain-derived neurotrophic factor. (2003) Neuroscience 119(2): 365-375.

2. Kivipelto, M., Laakso, M.P., Tuomilehto, J., et al. Hypertension and hypercholesterolaemia as risk factors for Alzheimer's disease: potential for pharmacological intervention. (2002) CNS Drugs 16(7): 435-444.

3. Lindqvist, A., Mohapel, P., Bouter, B., et al. High fat diet impairs hippocampal neurogenesis in male rats. (2006) Eur J Neurol 13(12): 1385-1388.

4. Solomon A., Kivipelto M, Wolozin, B., et al. Mid life serum cholesterol and increased risk of Alzheimers disease and vascular dementia three decades later. (2009) Dement Geriatr Cogn Disord 28(1): 75-80.

5. Sunanda, B.S., Shankaranarayana, R., Raju, T.R. Chronic restraint stress impairs acquisition and retention of spatial memory task in rats. (2000) Current Science 79: 1581-1584.

6. Smith, M.A., Makino, S., Kvetnansky, R., et al. Post. Stress and Glucocorticoids Affect the Expression of Brain-Derived Neurotrophic Factor and Neurotrophin-3 mRNAs in the Hippocampus. (1995) J Neurosci 15(3): 1766-1777.
7. Baran, S.E., Campbell, A.M., Kleen, J.K., et al. combination of high fat and chronic stress retracts hippocampal dendrites. (2005) Neuroreport 16(1): 39-43.

8. Vishal, R.T., Gupta, B.M., Ritu, T. Non-lipid actions of statins. (2004) jk science 3: 124-126.

9. Jick, H., Zornberg, G.L., Jick, S.S., et al. Statins and the risk of dementia. (2000) Lancet. 356(9242): 1627-1631.

10. Sparks, D.L., Sabbgh, M.N., Connor, D.J., et al. Atorvastatin for the treatment of mild to moderate Alzheimer's disease: preliminary results. (2005) Arch Neurol 62(5): 753-757.

11.Vishnu Kumar, Sushma Singh, Ashok Kumar Khanna et al. Hypoliedimic activity of anthocephalus indicus (kadam) in hyperlidemic rats. (2008) Med Chem Res 17(2-7): 152-158.

12. Shankaranarayana Rao, B. S., Madhavi, R., Sunanda et al. Complete reversal of dendritic atrophy in CA3 neurons of hippocampusby rehabilitation in restraint stressed rats. (2001) Current Science 80(5): 653-659.

13. Georgieva-Kotetarova, M.T., Kostadinova, I.I. Effect of atorvastatin and rosuvastatin on learning and memory in rats with diazepam-induced amnesia. (2013) Folia Medica 55(2): 58-65.

14. Luine, V., Villegas, M., Martinez, C., et al. Repeated stress causes reversible impairments of spatial memory performance. (1994) Brain Research 639(1): 167-170.

15. Arun Kumar Dubey, Ahalya Devi, Gopalan Kutty et al. Hypolipidemic Activity of Ginkgo biloba Extract, EGb 761 in HypercholesterolemicWistarRats. (2005) Iranian J Pharma \&Therapeutics 4(1): 9-12.

16. Madhyastha,S., Somayaji,S.N., Bairy, K.L., et al. Neuroprotective Effect of Centellaasiatica leaf extract treatment on cognition and hippocampal morphology against prenatal stress. (2007) Thai J Physiological Sciences 20(2): 79-88.

17. Gavin, J. B., Paul, M. R. Are statins anti-inflammatory? (2000) Curr Control Trials Cardiovasc Med 1(3): 161-165.

18. Broncel, M., Koter-Michalak, M., Chojnowska-Jezierska, J. The effect of statins on lipids peroxidation and activities of antioxidants enzymes in patients with dyslipidemia. (2006) Przegl Lek 63(9):738-742. 19. Parle,M., Singh, N. Reversal of memory deficits by atorvastatin and simvastatin in rats. (2002) Yaku Gaku Zasshi 127(7): 1125-1137.

20. Wolozin, B., Kellman,W., Ruosseau,P., et al. decreased prevalence of Alzhemiers disease associated with 3-hydroxy 3-methylglutaryl coenzyme A reductase inhibitors. (2005) Arch Neurology 57(10):14391443.

21. Rech, R.L., de Lima, M.N., Dornelles, A., et al. Reversal of age-associated memory impairment by rosuvastatin in rats. (2010) Exp Gerontol 45(5):351-356.

22. Georgieva-Kotetarova, M.T., Kostadinova, I.I. Effect of atorvastatin and rosuvastatin on learning and memory In rats with diazepam-induced amnesia. (2013) Folia Medica 55(2): 58-65.

23. Erdos, B., Snipes, J.A., Miller, A.W., et al. Cerebrovascular dysfunction in Zucker obese rats is mediated by oxidative stress and protein kinase C. (2004) Diabetes 53(5): 1352-1359.

24. Atsushi, I. Asymmetry of Symptoms and Signs in Multiple System Atrophy (2014)Int J Neurol Brain Disord 1(1): 1-2.

25. Vanita, P., et al. Screening of Wilson's Diseases and effect on the Brain at Exon 8. (2014) Int J Neurol Brain Disord 1(1): 1-15.

26. Severn, B.C., et al. Traumatic Brain Injury Causes a Tacrolimus-Sensitive Increase in Non-Convulsive Seizures in a Rat Model of Post-Traumatic Epilepsy (2014) Int J Neurol Brain Disord 1(1): 1-11. 